Turk. J. Math. Comput. Sci.

13(2)(2021) 331-337

(C) MatDer

DOI : $10.47000 /$ tjmcs.950099

\title{
On Involute-Evolute Curve Pair in Semi-Euclidean Space
}

\author{
Tuba AĞIRman Aydin ${ }^{1, *}$ (iD, Hüseyin KocaYigiT ${ }^{2}$ \\ ${ }^{1}$ Department of Maths, Faculty of Education, Bayburt University, 69000, Bayburt, Turkey. \\ ${ }^{2}$ Department of Maths, Faculty of Science and Arts, Celal Bayar University, 45000, Manisa, Turkey.
}

Received: 09-06-2021 • Accepted: 25-10-2021

\begin{abstract}
In this study, a kind of generalized involute and evolute curve pair is considered in 4 dimensional semi Euclidean space with 2 index. The curvatures and the associated Frenet Frame of this kind of generalized involute-evolute curve pair are presented.
\end{abstract}

2010 AMS Classification: 53A04, 53B30, 53A35

Keywords: Semi-Euclidean space, timelike curves, involute, evolute, curve pairs.

\section{INTRODUCTION}

Different curve couples are defined by examining the situations of the Frenet vectors of the curves relative to each other at their corresponding points. Involute-evolute curve pair is one of them. The involute-evolute curve pair were discovered by Huggens while trying to build a more accurate clock [2]. Fuchs defined the involute of a given curve as a curve in such the tangent vector of the given curve at each point corresponds its normal vector [5]. The involute-evolute curve pair is well known in 3-dimensional Euclidean space [6,11]. The relations Frenet vectors of involute-evolute curve pair were given in $E^{3}$ [7]. Turgut and Erdoğan examined this curve pair in $E^{n}$ [16]. Özyılmaz and Yılmaz determined that an evolute Frenet apparatus can be formed by an involute apparatus in four dimensional Euclidean space, so, in this way, another orthonormal frame in the same space can be obtained [14].

On the other hand, this curve pair has been studied by many researchers in Minkowski space [10,12]. Bükçü and Karacan presented the involute-evolute curves of the spacelike curve with a spacelike principal normal in Minkowski 3-Space [3]. Hanif et al. presented a special kind of generalized involute and evolute curve pair in 4-dimensional Minkowski space by considering this curve pair in a different way. [9].

In this article, we consider $(0,2)$-involute curve of a given timelike curve with respect to the causal characteristics of the (0,2)-plane that is spanned by the tangent and first binormal of the vector fields in the $R_{2}^{4}$. Similarly, we examine $(1,3)$-evolute curve of a given timelike curve with respect to the causal characteristics of the $(1,3)$-normal plane that are spanned by the principal normal and the second binormal of the vector fields in the same space.

*Corresponding Author

Email addresses: tubagirman@hotmail.com (T. Ağırman Aydın), huseyin.kocayigit@cbu.edu.tr (H. Kocayiğit) 


\section{Preliminaries}

To meet the requirements in the next sections, the basic elements of the theory of curves in semi-Euclidean space $R_{2}^{4}$ are briefly presented in this section.

Let $R_{2}^{4}$ denote 4-dimensional semi-Euclidean space with 2-index provided with standard flat metric given by

$$
g=-d a_{1}^{2}-d a_{2}^{2}+d a_{3}^{2}+d a_{4}^{2},
$$

where $\left(a_{1}, a_{2}, a_{3}, a_{4}\right)$ is a rectangular coordinate system of $R^{4}$. A vector $w$ in $R_{2}^{4}$ is called a spacelike, timelike or null (lightlike) if hold $g(w, w)>0, g(w, w)<0$ or $g(w, w)=0$ and $w \neq 0$, respectively. The norm of a vector $w$ is given by $\|w\|=\sqrt{|g(w, w)|}$. Therefore, $w$ is a unit vector if $g(w, w)= \pm 1$. Two vectors $u$ and $w$ are said to be orthogonal if $g(u, w)=0$ [13]. Also, let $u$ and $w$ be two timelike vectors in $R_{2}^{4}$. An arbitrary curve $\gamma=\gamma(s)$ in $R_{2}^{4}$ can locally be spacelike, timelike or null (lightlike) if all of its velocity vectors $\gamma^{\prime}(s)$ are, respectively, spacelike, timelike or null (lightlike). The velocity of the curve $\gamma$ is given by $\left\|\gamma^{\prime}\right\|$. Thus, a timelike curve $\gamma$ is said to be parametrized by arc length function $s$ if $g\left(\gamma^{\prime}, \gamma^{\prime}\right)=-1[13]$.

Let $\left\{T(s), N(s), B_{1}(s), B_{2}(s)\right\}$ denotes the moving Frenet frame along $\gamma$ in the semi-Euclidean space $R_{2}^{4}$, then $T(s), N(s), B_{1}(s)$ and $B_{2}(s)$ are called the tangent, the principal normal, the first binormal, and the second binormal vector fields of $\gamma$, respectively.

A unit speed curve $\gamma$ is said to be a Frenet curve if $g\left(\gamma^{\prime \prime}, \gamma^{\prime \prime}\right) \neq 0$. Let $\gamma$ be a $C^{\infty}$ special timelike Frenet curve with timelike principal normal, spacelike both first binormal and second binormal vector fields in $R_{2}^{4}$, parametrized by arc length function $s$. Moreover, non-zero $C^{\infty}$ scalar functions $\kappa_{1}, \kappa_{2}$ and $\kappa_{3}$ be the first, second, and third curvatures of $\gamma$, respectively. Then for a $C^{\infty}$ special nonnull Frenet curve $\gamma$, the Frenet formula is given by

$$
\begin{aligned}
T^{\prime} & =\varepsilon \kappa_{1} N \\
N^{\prime} & =-\varepsilon \kappa_{1} T+\kappa_{2} B_{1} \\
B_{1}^{\prime} & =\kappa_{2} N+\kappa_{3} B_{2} \\
B_{2}^{\prime} & =-\kappa_{3} B_{1},
\end{aligned}
$$

where $T, N, B_{1}$ and $B_{2}$ mutually orthogonal vector fields satisfying

$$
g(T, T)=g(N, N)=\varepsilon, g\left(B_{1}, B_{1}\right)=g\left(B_{2}, B_{2}\right)=-\varepsilon
$$

and $\varepsilon=\left\{\begin{array}{c}+1 ; \gamma \text { spacelike } \\ -1 ; \gamma \text { timelike }\end{array}\right.$ (for the semi-Euclidean space $E_{v}^{n+1}$, see $[1,4,15]$ ).

Definition 2.1 ( [3]). Let any curves $\gamma$ and $\gamma^{*}$ be given with coordinate neighborhoods $(I, \gamma)$ and $\left(I, \gamma^{*}\right)$, respectively. Let's denote the Frenet frames at the points $\gamma(s), \gamma^{*}(s)$ corresponding to $s$ by $\{T, N, B\}$ and $\left\{T^{*}, N^{*}, B^{*}\right\}$, respectively. If $\mathrm{g}\left(T, T^{*}\right)=0$ for $\forall s \in I$, the curve pair $\left(\gamma, \gamma^{*}\right)$ is the involute-evolute curve pair. Then the curve $\gamma^{*}$ is called the involute of the curve $\gamma$ and the curve $\gamma$ is called the evolute of the curve $\gamma^{*}$ [16].

Definition 2.2 ( [3]). The plane spanned by $\left\{T, B_{1}\right\}$ is called the $(0,2)$-tangent plane of $\gamma$ at any point of $\gamma$, . The plane spanned by $\left\{N, B_{2}\right\}$ is called the $(1,3)$-normal plane of $\gamma[8]$.

Definition 2.3 ( [3]). Let $\gamma: I \rightarrow R_{2}^{4}$ and $\gamma^{*}: I \rightarrow R_{2}^{4}$ be two regular curves in $R_{2}^{4}$, where $s$ is the arc-length parameter of $\gamma$. Denote $s^{*}=\varphi(s)$ to be the arc-length parameters of $\gamma^{*}$. For $\forall s \in I$, if the $(0,2)$-tangent plane of $\gamma$ at $\gamma(s)$ of coincides with the $(1,3)$-normal plane at $\gamma^{*}(s)$ of $\gamma^{*}$, then $\gamma^{*}$ is called the $(0,2)$-involute curve of $\gamma$ and $\gamma$ is called the $(1,3)$-evolute curve of $\gamma^{*}$ in the $R_{2}^{4}$.

\section{The $(0,2)$-Involute Curve of a Timelike Curve in $E_{2}^{4}$}

In this section, we proceed to study the existence and expression of the $(0,2)$-involute curve of a given timelike curve in $R_{2}^{4}$.

Let $\gamma: I \rightarrow R_{2}^{4}$ be a regular timelike curve with arc-length parameter $s$ and $\kappa_{1}, \kappa_{2}$ and $\kappa_{3}$ never vanish. Suppose that $\gamma^{*}: I \rightarrow R_{2}^{4}$ is the $(0,2)$-involute curve of $\gamma$. It is clear that $\gamma^{*}$ can be timelike or spacelike. Denote $T^{*}, N^{*}, B_{1}^{*}, B_{2}^{*}$ to be the Frenet frame along and $\kappa_{1}^{*}, \kappa_{2}^{*}$ and $\kappa_{3}^{*}$ to be the curvatures of $\gamma^{*}$. Then

$$
\operatorname{span}\left\{T, B_{1}\right\}=\operatorname{span}\left\{N^{*}, B_{2}^{*}\right\}, \operatorname{span}\left\{N, B_{2}\right\}=\operatorname{span}\left\{T^{*}, B_{1}^{*}\right\} \text {. }
$$


Moreover, $\gamma^{*}$ can be expressed as

$$
\gamma^{*}(s)=\gamma(s)+\lambda(s) T(s)+\mu(s) B_{1},
$$

where $\lambda(s)$ and $\mu(s)$ are $C^{\infty}$ functions on $I$. Differentiating (3.1) with respect to $s$ and using the Frenet formula (2.1), we get

$$
\varphi^{\prime} T^{*}=\left(1+\lambda^{\prime}\right) T+\left(\mu \kappa_{2}-\lambda \kappa_{1}\right) N+\mu^{\prime} B_{1}+\mu \kappa_{3} B_{2} .
$$

By the inner products of both-sides of (3.2) with $T$ and $B_{1}$, respectively, we get $1+\lambda^{\prime}=0$ and $\mu^{\prime}=0$, which implies that $\mu$ is constant and $\lambda=\lambda_{0}-s$, where $\lambda_{0}$ is the integration constant and $\varphi^{\prime}=d s^{*} / d s$. So (3.2) becomes

$$
\varphi^{\prime} T^{*}=\left(\mu \kappa_{2}-\lambda \kappa_{1}\right) N+\mu \kappa_{3} B_{2} .
$$

Denote

Then (3.3) can be written as

$$
\delta=\frac{\mu \kappa_{2}-\lambda \kappa_{1}}{\varphi^{\prime}}, \zeta=\frac{\mu \kappa_{3}}{\varphi^{\prime}} .
$$

$$
T^{*}=\delta N+\zeta B_{2}, \zeta^{2}-\delta^{2}=\varepsilon
$$

Case 1: $\mu \neq 0$. In this case, $\zeta \neq 0$. Denote $\delta / \zeta=\alpha_{1}$. Then $\delta=\zeta \alpha_{1}$ and

$$
\mu \kappa_{2}-\lambda \kappa_{1}=\alpha_{1} \mu \kappa_{3}, \varphi^{\prime}=\zeta^{-1} \mu \kappa_{3}, \zeta^{2}=\frac{\varepsilon}{1-\alpha_{1}^{2}} .
$$

Differentiating (3.4) with respect to $s$ and using the Frenet frame (2.1) we get

$$
\varepsilon \varphi^{\prime} \kappa_{1}^{*} N^{*}=\delta \kappa c_{1} T+\delta^{\prime} N+\left(\delta \kappa_{2}-\zeta \kappa_{3}\right) B_{1}+\zeta^{\prime} B_{2}
$$

By the inner products of both-sides of (3.6) with $N$ and $B_{2}$, respectively, we get $\delta^{\prime}=0$ and $\zeta^{\prime}=0$, which implies that $\delta$ and $\zeta$ are constants. So (3.6) gives us

$$
\varepsilon \varphi^{\prime} \kappa_{1}^{*} N^{*}=\delta \kappa_{1} T+\left(\delta \kappa_{2}-\zeta \kappa_{3}\right) B_{1} .
$$

Denote

$$
\vartheta=\frac{\zeta \alpha_{1} \kappa_{1}}{\varepsilon \varphi^{\prime} \kappa_{1}^{*}}, \varrho=\frac{\zeta\left(\alpha_{1} \kappa_{2}-\kappa_{3}\right)}{\varepsilon \varphi^{\prime} \kappa_{1}^{*}} .
$$

So from the equation (3.7), we get

$$
N^{*}=\vartheta T+\varrho B_{1}, \varepsilon=\varrho^{2}-\vartheta^{2} .
$$

Denote $\varrho / \vartheta=\alpha_{2}$. Then we find $\varrho=\alpha_{2} \vartheta$ and

$$
\alpha_{1}\left(\kappa_{2}-\alpha_{2} \kappa_{1}\right)=\kappa_{3}, \vartheta^{2}=\frac{\varepsilon}{\alpha_{2}^{2}-1} .
$$

From the first equations of (3.5) and (3.10), we have

$$
\eta=\frac{\kappa_{2}}{\kappa_{1}}=\frac{\lambda / \mu-\alpha_{1}^{2} \alpha_{2}}{1-\alpha_{1}^{2}}, \frac{\kappa_{3}}{\kappa_{1}}=\alpha_{1}\left(\eta-\alpha_{2}\right) .
$$

Denote $\zeta / \vartheta=\alpha_{3}$. Then $\zeta=\alpha_{3} \vartheta$. From (3.8), we have

$$
\varepsilon \varphi^{\prime} \kappa_{1}^{*}=\alpha_{1} \alpha_{3} \kappa_{1}, \alpha_{3}^{2}=\frac{\alpha_{2}^{2}-1}{1-\alpha_{1}^{2}} .
$$

Differentiating (3.9) with respect to $s$ and using the Frenet formula, we get

$$
-\varepsilon \varphi^{\prime} \kappa_{1}^{*} T^{*}+\varphi^{\prime} \kappa_{2}^{*} B_{1}^{*}=\vartheta^{\prime} T+\left(\varrho \kappa_{2}-\vartheta \kappa_{1}\right) N+\varrho^{\prime} B_{1}+\varrho \kappa_{3} B_{2} .
$$

By the inner products of both-sides of (3.12) with $T$ and $B_{1}$, respectively, we get $\vartheta^{\prime}=0$ and $\varrho^{\prime}=0$, which implies that $\vartheta$ and $\varrho$ are constants. In this case, (3.12) turns into

$$
\varphi^{\prime} \kappa_{2}^{*} B_{1}^{*}=\left(\varrho \kappa_{2}-\vartheta \kappa_{1}\right) N+\varrho \kappa_{3} B_{2}+\varepsilon \varphi^{\prime} \kappa_{1}^{*} T^{*} .
$$

Substituting (3.4) and (3.11) into (3.13), we obtain

$$
\varphi^{\prime} \kappa_{2}^{*} B_{1}^{*}=\vartheta \kappa_{1}\left(\alpha_{2} \eta+\alpha_{3}^{2}-\alpha_{2}^{2}\right)\left(N+\alpha_{1} B_{2}\right) .
$$

From (3.14), we may choose that

$$
B_{1}^{*}=\zeta N+\delta B_{2}, \varphi^{\prime} \kappa_{2}^{*}=\alpha_{3}^{-1} \kappa_{1}\left(\alpha_{2} \eta+\alpha_{3}^{2}-\alpha_{2}^{2}\right) .
$$


Differentiating with respect to $s$ and using the Frenet formula (2.1), we get

$$
\varphi^{\prime} \kappa_{2}^{*} N^{*}+\varphi^{\prime} \kappa_{3}^{*} B_{2}^{*}=\zeta \kappa_{1} T+\left(\zeta \kappa_{2}-\delta \kappa_{3}\right) B_{1},
$$

from which we obtain

$$
\varphi^{\prime} \kappa_{3}^{*} B_{2}^{*}=-\alpha_{3}^{-1} \kappa_{1}\left(\eta-\alpha_{2}\right)\left(\varrho T+\vartheta B_{1}\right)
$$

From (3.15), we may choose that

$$
B_{2}^{*}=\varrho T+\vartheta B_{1}, \varphi^{\prime} \kappa_{3}^{*}=-\alpha_{3}^{-1} \kappa_{1}\left(\eta-\alpha_{2}\right)
$$

So we can give the following theorem.

Theorem 3.1. Let $\gamma: I \rightarrow R_{2}^{4}$ be a regular timelike curve with arc-length parameter s so that $\kappa_{1}$, $\kappa_{2}$ and $\kappa_{3}$ never vanish. If $\gamma^{*}: I \rightarrow R_{2}^{4}$ is the $(0,2)$-involute mate curve of $\gamma, \gamma^{*}(s)=\gamma(s)+\left(\lambda_{0}-s\right) T(s)+\mu B_{1}$ with $\mu \neq 0$, then $\kappa_{1}$, $\kappa_{2}$ and $\kappa_{3}$ satisfy

$$
\frac{\kappa_{2}}{\kappa_{1}}=\eta, \frac{\kappa_{3}}{\kappa_{1}}=\alpha_{1}\left(\eta-\alpha_{2}\right), \eta=\frac{\lambda_{0}-s-\mu \alpha_{1}^{2} \alpha_{2}}{\mu\left(1-\alpha_{1}^{2}\right)},
$$

where $\lambda_{0}, \mu, \alpha_{1}$ and $\alpha_{2}$ are given constants. Moreover, the three curvatures of $\gamma^{*}$ are given by

$$
\kappa_{1}^{*}=\frac{\vartheta \alpha_{3}^{2}}{\varepsilon \mu\left(\eta-\alpha_{2}\right)}, \kappa_{2}^{*}=\frac{\vartheta\left(\alpha_{2} \eta+\alpha_{3}^{2}-\alpha_{2}^{2}\right)}{\mu \alpha_{1}\left(\eta-\alpha_{2}\right)}, \kappa_{3}^{*}=-\frac{\vartheta}{\mu \alpha_{1}},
$$

where $\vartheta \neq 0$. The associated Frenet Frame are given by

$$
T^{*}=\vartheta \alpha_{3}\left(\alpha_{1} N+B_{2}\right), N^{*}=\vartheta\left(T+\alpha_{2} B_{1}\right), B_{1}^{*}=\vartheta \alpha_{3}\left(N+\alpha_{1} B_{2}\right), B_{2}^{*}=\vartheta\left(\alpha_{2} T+B_{1}\right) .
$$

Case 2: $\mu=0$. In this case, (3.1) turns into

$$
\gamma^{*}(s)=\gamma(s)+\left(\lambda_{0}-s\right) T(s) .
$$

Differentiating (3.16) with respect to $s$ and using the Frenet formula (2.1), we get

$$
\varphi^{\prime} T^{*}=-\left(\lambda_{0}-s\right) \kappa_{1} N
$$

from which we may assume that

$$
\varphi^{\prime}=\left(\lambda_{0}-s\right) \kappa_{1}, T^{*}=-N .
$$

Differentiating the second equation of (3.17) with respect to $s$ and using the Frenet formula (2.1), we get

$$
\varepsilon \varphi^{\prime} \kappa_{1}^{*} N^{*}=-\kappa_{1} T-\kappa_{2} B_{1} \text {. }
$$

Suppose that

$$
N^{*}=\vartheta T+\varrho B_{1}, \vartheta=\frac{-\kappa_{1}}{\varepsilon \varphi^{\prime} \kappa_{1}^{*}}, \varrho=\frac{-\kappa_{2}}{\varepsilon \varphi^{\prime} \kappa_{1}^{*}},-\vartheta^{2}+\varrho^{2}=\varepsilon
$$

It follows that

$$
\frac{\kappa_{2}}{\kappa_{1}}=\frac{\varrho}{\vartheta} \text {. }
$$

Differentiating (3.18) with respect to $s$, we obtain that $\vartheta$ and $\varrho$ are constant and

Suppose that

$$
\varphi^{\prime} \kappa_{2}^{*} B_{1}^{*}=\varepsilon \varphi^{\prime} \kappa_{1}^{*} T^{*}+\left(-\vartheta \kappa_{1}+\varrho \kappa_{2}\right) N+e \kappa_{3} B_{2}=-\frac{\kappa_{1}}{\vartheta}(1+\varepsilon) N+\varrho \kappa_{3} B_{2} .
$$

$$
B_{1}^{*}=B_{2}, \varphi^{\prime} \kappa_{2}^{*}=\varrho \kappa_{3}, \frac{\kappa_{1}}{\vartheta}(1+\varepsilon)=0 .
$$

Corollary 3.2. Since $\kappa_{1} \neq 0, \varepsilon=-1$ in the last equation of expression (3.19), the (0,2)-involute mate curve of a timelike curve can only be timelike, for $\mu=0$.

Differentiating (3.19) with respect to $s$, we obtain

$$
\varphi^{\prime} \kappa_{3}^{*} B_{2}^{*}=-\varphi^{\prime} \kappa_{2}^{*} N^{*}-\kappa_{3} B_{1}=-\vartheta \kappa_{3}\left(\varrho T+\vartheta B_{1}\right) .
$$

We suppose that $T^{*} \times N^{*} \times B_{1}^{*} \times B_{2}^{*}=T \times N \times B_{1} \times B_{2}$. Then

$$
B_{2}^{*}=\varrho T+\vartheta B_{1}, \varphi^{\prime} \kappa_{3}^{*}=-\vartheta \kappa_{3} .
$$

So we can give the following theorem. 
Theorem 3.3. Let $\gamma: I \rightarrow R_{2}^{4}$ be a regular timelike curve with arc-length parameter $s$ so that $\kappa_{1}, \kappa_{2}$ and $\kappa_{3}$ never vanish. If $\gamma^{*}: I \rightarrow R_{2}^{4}$ is the $(0,2)$-involute mate curve of $\gamma, \gamma^{*}(s)=\gamma(s)+\left(\lambda_{0}-s\right) T(s)$ with $\mu=0$, then $\kappa_{1}$, $\kappa_{2}$ satisfy

$$
\varrho \kappa_{1}-\vartheta \kappa_{2}=0
$$

where $\lambda_{0}, \vartheta$ and $\varrho$ are given constants. Moreover, the three curvatures of $\gamma^{*}$ are given by

The associated Frenet Frame are given by

$$
\kappa_{1}^{*}=\frac{-1}{\varepsilon\left(\lambda_{0}-s\right) \vartheta}, \kappa_{2}^{*}=\frac{\varrho \kappa_{3}}{\left(\lambda_{0}-s\right) \kappa_{1}}, \kappa_{3}^{*}=\frac{-\vartheta \kappa_{3}}{\left(\lambda_{0}-s\right) \kappa_{1}} .
$$

$$
T^{*}=-N, N^{*}=\vartheta T+\varrho B_{1}, B_{1}^{*}=B_{2}, B_{2}^{*}=\varrho T+\vartheta B_{1} .
$$

\section{The $(1,3)$-Evolute Curve of a Timelike Curve in $E_{2}^{4}$}

In this section, we proceed to study the existence and expression of the (1,3)-evolute curve of a given timelike curve in $R_{2}^{4}$.

Let $\gamma: I \rightarrow R_{2}^{4}$ be a regular timelike curve with arc-length parameter $s$ so that $\kappa_{1}, \kappa_{2}$ and $\kappa_{3}$ never vanish. Suppose that $\gamma^{*}: I \rightarrow R_{2}^{4}$ is the (1,3)-evolute curve of $\gamma$. It is clear that $\gamma^{*}$ can be timelike or spacelike. Denote $T^{*}, N^{*}, B_{1}^{*}, B_{2}^{*}$ to be the Frenet frame along and $\kappa_{1}^{*}, \kappa_{2}^{*}$ and $\kappa_{3}^{*}$ to be the curvatures of $\gamma^{*}$. Then

$$
\operatorname{span}\left\{T, B_{1}\right\}=\operatorname{span}\left\{N^{*}, B_{2}^{*}\right\}, \operatorname{span}\left\{N, B_{2}\right\}=\operatorname{span}\left\{T^{*}, B_{1}^{*}\right\}, T \perp T^{*} .
$$

Moreover, $\gamma^{*}$ can be expressed as

$$
\gamma^{*}(s)=\gamma(s)+v(s) N(s)+w(s) B_{2},
$$

where $v(s)$ and $w(s)$ are $C^{\infty}$ functions on $I$. Differentiating (4.1) with respect to $s$ and using the Frenet formula (2.1), we get

$$
\varphi^{\prime} T^{*}=\left(1+v \kappa_{1}\right) T+v^{\prime} N+\left(v \kappa_{2}-w \kappa_{3}\right) B_{1}+w^{\prime} B_{2} .
$$

By the inner products of both-sides of (4.2) with $T$ and $B_{1}$, respectively, we get

$$
\varphi^{\prime} T^{*}=v^{\prime} N+w^{\prime} B_{2}, v=-\frac{1}{\kappa_{1}}, w=-\frac{\kappa_{2}}{\kappa_{1} \kappa_{3}} .
$$

Denote

Then (4.3) turns into

$$
a=\frac{v^{\prime}}{\varphi^{\prime}}, b=\frac{w^{\prime}}{\varphi^{\prime}} .
$$

$$
T^{*}=a N+b B_{2}, b^{2}-a^{2}=\varepsilon .
$$

Differentiating (4.5) with respect to $s$ and using the Frenet formula (2.1), we get

$$
\varepsilon \varphi^{\prime} \kappa_{1}^{*} N^{*}=a \kappa_{1} T+a^{\prime} N+\left(a \kappa_{2}-b \kappa_{3}\right) B_{1}+b^{\prime} B_{2} .
$$

Taking inner product on both-sides of (4.6) with $N$ and $B_{2}$ respectively, we get $a^{\prime}=0$ and $b^{\prime}=0$ which implies that $a$ and $b$ are constants. From (4.4), we obtain

$$
v=a \varphi+v_{0}=-\frac{1}{\kappa_{1}}, w=b \varphi+w_{0}=-\frac{\kappa_{2}}{\kappa_{1} \kappa_{3}} .
$$

Moreover, (4.6) turns into

Denote

$$
\varepsilon \varphi^{\prime} \kappa_{1}^{*} N^{*}=a \kappa_{1} T+\left(a \kappa_{2}-b \kappa_{3}\right) B_{1} .
$$

Then (4.8) turns into

$$
m=\frac{a \kappa_{1}}{\varepsilon \varphi^{\prime} \kappa_{1}^{*}}, n=\frac{a \kappa_{2}-b \kappa_{3}}{\varepsilon \varphi^{\prime} \kappa_{1}^{*}} .
$$

$$
N^{*}=m T+n B_{1}, \varepsilon \varphi^{\prime} \kappa_{1}^{*}=m^{-1} a \kappa_{1}, n^{2}-m^{2}=\varepsilon
$$

Moreover, we have

$$
n a \kappa_{1}+m b \kappa_{3}-m a \kappa_{2}=0
$$

Case 1: $n \neq 0$. Differentiating (4.9) with respect to $s$ and using (2.1), we obtain

$$
-\varepsilon \varphi^{\prime} \kappa_{1}^{*} T^{*}+\varphi^{\prime} \kappa_{2}^{*} B_{1}^{*}=m^{\prime} T+\left(n \kappa_{2}-m \kappa_{1}\right) N+n^{\prime} B_{1}+n \kappa_{3} B_{2} .
$$


By the inner products of both-sides of (4.10) with $T$ and $B_{1}$ respectively, we get $m^{\prime}=0$ and $n^{\prime}=0$, which implies that $m$ and $n$ are constants. In this case, (4.10) turns into

$$
\varphi^{\prime} \kappa_{2}^{*} B_{1}^{*}=\left(\frac{a^{2}-m^{2}}{m} \kappa_{1}+n \kappa_{2}\right) N+\left(\frac{a b}{m} \kappa_{1}+n \kappa_{3}\right) B_{2} .
$$

Denote

Then (4.11) turns into

$$
\psi=\left(\varphi^{\prime} \kappa_{2}^{*}\right)^{-1}\left(\frac{a^{2}-m^{2}}{m} \kappa_{1}+n \kappa_{2}\right), \omega=\left(\varphi^{\prime} \kappa_{2}^{*}\right)^{-1}\left(\frac{a b}{m} \kappa_{1}+n \kappa_{3}\right)
$$

$$
B_{1}^{*}=\psi N+\omega B_{2}, \omega^{2}-\psi^{2}=\varepsilon
$$

Since $T^{*} \perp B_{1}^{*}$, it follows from (4.5) and (4.12) that $\psi / \omega=b / a$, which implies that

$$
B_{1}^{*}=b N+a B_{2}, \varphi^{\prime} \kappa_{2}^{*}=\frac{b \kappa_{1}}{m}+\frac{n \kappa_{3}}{a} .
$$

Differentiating (4.13) with respect to $s$ and using (2.1), we get

$$
\varphi^{\prime} \kappa_{3}^{*} B_{2}^{*}=-\varphi^{\prime} \kappa_{2}^{*} N^{*}+b \kappa_{1} T+\left(b \kappa_{2}-a \kappa_{3}\right) B_{1}=-\frac{m \kappa_{3}}{a}\left(n T+m B_{1}\right) .
$$

It follows that from (4.14) that

So we can give the following theorem.

$$
B_{2}^{*}=n T+m B_{1}, \varphi^{\prime} \kappa_{3}^{*}=-\frac{m \kappa_{3}}{a} .
$$

Theorem 4.1. Let $\gamma: I \rightarrow R_{2}^{4}$ be a regular curve with arc-length parameter s so that $\kappa_{1}, \kappa_{2}$ and $\kappa_{3}$ never vanish. Suppose that $\gamma^{*}: I \rightarrow R_{2}^{4}$ the $(1,3)$-evolute mate curve of $\gamma$,

$$
\gamma^{*}(s)=\gamma(s)-\frac{1}{a \kappa_{1}}\left[a N(s)+b B_{2}\right]-\frac{n}{m \kappa_{3}} B_{2},
$$

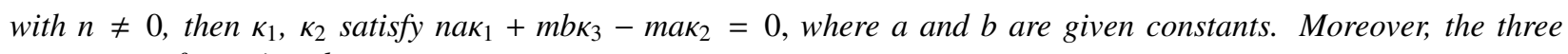
curvatures of are given by

The associated Frenet Frame are given by

$$
\kappa_{1}^{*}=\frac{a \kappa_{1}}{\varepsilon \varphi^{\prime} m}, \kappa_{2}^{*}=\frac{b \kappa_{1}}{\varphi^{\prime} m}+\frac{n \kappa_{3}}{\varphi^{\prime} a}, \kappa_{3}^{*}=-\frac{m \kappa_{3}}{\varphi^{\prime} a} .
$$

$$
T^{*}=a N+b B_{2}, N^{*}=m T+n B_{1}, B_{1}^{*}=b N+a B_{2}, B_{2}^{*}=n T+m B_{1} .
$$

Case 2: $n=0$. In this case, we may suppose that

$$
N^{*}=m T, \varepsilon \varphi^{\prime} \kappa_{1}^{*}=m^{-1} a \kappa_{1}, a \kappa_{2}-b \kappa_{3}=0, \varepsilon=-m^{2} .
$$

Corollary 4.2. Since $m^{2}=-\varepsilon, \varepsilon \leq 0$, the $(1,3)$-evolute mate curve of a timelike curve in $R_{2}^{4}$ can only be timelike, for $n=0$. Also $m=\varepsilon$.

Moreover, we have from (4.7) and the third equation of (4.15) that

$$
v=a\left(\varphi+\varphi_{0}\right)=-\frac{1}{\kappa_{1}}, w=b\left(\varphi+\varphi_{0}\right)=-\frac{b}{a \kappa_{1}} .
$$

Differentiating (4.15) with respect to $s$ and using (2.1), we get

$$
\varphi^{\prime} \kappa_{2}^{*} B_{1}^{*}=\varepsilon \varphi^{\prime} \kappa_{1}^{*} T^{*}-m \kappa_{1} N
$$

It follows that we may choose

$$
B_{1}^{*}=b N+a B_{2}, \varphi^{\prime} \kappa_{2}^{*}=\frac{b \kappa_{1}}{\varepsilon} .
$$

Differentiating (4.16) with respect to $s$, using (2.1) and the third equation of (4.15), we get

$$
\varphi^{\prime} \kappa_{3}^{*} B_{2}^{*}=-\varphi^{\prime} \kappa_{2}^{*} N^{*}+\left(b \kappa_{2}-a \kappa_{3}\right) B_{1} .
$$

from which we obtain

$$
B_{2}^{*}=m B_{1}, \varphi^{\prime} \kappa_{3}^{*}=a^{-1} \kappa_{3}
$$

So we can give the following theorem. 
Theorem 4.3. Let $\gamma: I \rightarrow R_{2}^{4}$ be a regular curve with arc-length parameter $s$ so that $\kappa_{1}, \kappa_{2}$ and $\kappa_{3}$ are not zero. Suppose that $\gamma^{*}: I \rightarrow R_{2}^{4}$ the (1,3)-evolute mate curve of $\gamma$,

$$
\gamma^{*}(s)=\gamma(s)-\frac{1}{a \kappa_{1}}\left[a N(s)+b B_{2}\right]
$$

with $a=0$, then $\kappa_{1}, \kappa_{2}$ satisfy $a \kappa_{2}-b \kappa_{3}=0$ where $a$ and $b$ are given constants. Moreover, the three curvatures of are given by

The associated Frenet Frame are given by

$$
\varphi^{\prime} \kappa_{1}^{*}=\frac{a \kappa_{1}}{\varphi^{\prime}}, \kappa_{2}^{*}=\frac{b \kappa_{1}}{\varepsilon \varphi^{\prime}}, \kappa_{3}^{*}=\frac{\kappa_{3}}{a \varphi^{\prime}} .
$$

$$
T^{*}=a N+b B_{2}, N^{*}=m T, B_{1}^{*}=b N+a B_{2}, B_{2}^{*}=m B_{1} .
$$

\section{RESUlts}

In this article a kind of generalized involute-evolute curve pair is presented in 4 dimensional semi Euclidean space with 2 index. We examine the existence of the (0,2)-involute curve and the (1,3)-evolute curve of the timelike curve for the different cases in the $R_{2}^{4}$. The curvatures and the associated Frenet Frame of this kind of generalized involuteevolute curve pair are presented for each existence state.

\section{CONFLICTS OF INTEREST}

The author/authors declare that there are no conflicts of interest regarding the publication of this article.

\section{Authors Contribution Statement}

All authors have contributed sufficiently in the planning, execution, or analysis of this study to be included as authors. All authors have read and agreed to the published version of the manuscript.

\section{REFERENCES}

[1] Akgun, M., Sivridağ, A.İ., Some characterizations for spacelike inclined curves, Commun. Fac. Sci. Univ. Ank. Ser. A1 Math. Stat., 68(2019), 1576-1585.

[2] Boyer, C., A History of Mathematics, New York, Wiley, 1968.

[3] Bükcü, B., Karacan, M.K., On the involute and evolute curves of the timelike curve in Minkowski 3-space, Demonstr. Math., 40(2007), 721-732.

[4] Çöken, A.C., Görgülü, A., On Joachimsthal's theorems in semi-Euclidean spaces, Nonlinear Analysis: Theory, Meth. App., 70(2009), 39323942.

[5] Fuchs, D., Evolutes and involutes of spatial curves, Am. Math. Mon., 120(2013), 217-231.

[6] Hacısalihoğlu, H.H., Yüksek Diferansiyel Geometriye Giriş, Fırat University Publications, Elazı̆̆ 1980.

[7] Hacisalihoglu, H.H., Diferansiyel Geometri, İnönü University Faculty of Arts and Sciences Publications, Malatya, 1983.

[8] Hanif, M., Hua Hou, Z., Generalized involute and evolute curve-couple in Euclidean space, Int. J. Open Problems Compt. Math., 11(2018), 28-39.

[9] Hanif, M., Hua Hou Z., Nisar, K.S., On special kinds of involute and evolute curves in 4-dimensional Minkowski space, Symmetry, 10(2018), 317.

[10] Izumiya, S., Takahashi, M., Spacelike parallels and evolutes in Minkowski pseudo-spheres, Journal of Geometry and Physics 57(2007).

[11] Millman, R.S., Parker, G.D., Elements of Differential Geometry, Prentice-Hall Inc., Englewood Cliffs, New Jersey, 1977.

[12] Nolasco, B., Pacheco, R., Evolutes of plane curves and null curves in Minkowski 3-space, J. Geom., 108(2017), 195-214.

[13] O’Neill, B., (1983). Semi-Riemannian Geometry with Applications to Relativity. Academic Press Inc., London.

[14] Özy1lmaz, E.,Yılmaz, S., Involute-evolute curve couples in the Euclidean 4-Space, Int. J. Open Probl.Compt. Math., 2(2009), $168-174$.

[15] Ersoy, S., Tosun, M., Timelike Bertrand curves in Semi-Euclidean space. Int. J. Math. Stat., 14(2013), 78-89.

[16] Turgut, A., Erdoğan, E., Involute evolute curve couples of higher order in $R^{n}$ and their horizontal lifts in $R^{n}$, Commun. Fac. Sci. Uni. Ankara, Ser. A, 41(1992), 125-130. 